\title{
BRAGG REFLECTION PROGRAM
}

\author{
User's Guide
}

J. W. Lynn

INFORMAL REPORT

NOTICE

This report was prepared as an account of work sponsored by the United States Government. Neither the U.S. Energy Research and Development Administration, nor sny of their employees, nor any of their contractors, subcontractors, or their employees, makes any warranty, express or Implied, oi assumes any legal liability or desponsibility for the accuracy, completeness or usefulness of any information, apparatus, product or process disclosed, or represents that its use would zut i-Eringe privately owned rights. 
BRAGG REFLECTION PROGRAM - User's Guide

The Bragg Refiection Program (BRP) for the PAP-1l system is analogous to the "PAIR" program for the old SDS system. Its general purpose is to automatically scan a series of Bragg reflections to obtain profiles and integrated intensities. The program is used in conjunction with the SUPERVISOR and READ packages and the procedures for using it are simflar to those for the Triple-Axis Control program. All the general features of the system, SUPERVISOR and READ packages as described in the Spectrometer Control System User's Gulde (SCSUG) have been preserved. To avoid lengthy duplication of material, it will be assumed that the reader is already familiar with these.

\section{$\underline{\text { Read Package }}$}

The general read varlables for the BRP are the same as those listed in Appendix $B$ of the Scsug except that six additional variables have been defincd. These varlables are treated fust the same as the other general read variables and do not change from scan to scan. They are defined as follows:

ST - Scan type. There are three typea of scan that can be performed by the program. With ST $=1$, the detector is moved to the scattering angle corresponding to $(\mathrm{H}, \mathrm{K})$, and the sample angle is scanned through the peak in steps $\Delta \psi$. With $S T=2$, a $\theta: 2 \theta$ scan is done. For $S T=3$, a $2 \theta$ scan is performed, with the sample angle FROZEN. In this mode the sample angle does not change efther during a scan or between scans, and the power to the motor may be shut off if desired.

If ST 1s not set to 1,2 or 3 , then the program w111 be ext.ted and an error message typed. 
Note on freezing motors: The sample angle must always be defined $(0 \leq \mathrm{C} 2<6)$. ST $=3$ provides for freezing the sample angle in the BRP itself. The scattering arm may be frozen v1a the READ package (1.e. A2 > 6) only when $S T=1$. Any other condition wIII abort the BRP and print an error message. All remaining angles may be either defined or undefined. The elastic condition $\mathrm{KI}=\mathrm{KF}$ is always assumed in BRP, and the angles which are defined for the monochromator or analyzer (depending on the value of IK) will be moved to the elastic position. DS - Step in angle $\Delta \psi$ for sample table when $S T=1$ or 2 ; step in $2 \theta$ when ST $=3$. DS can be positive, negative or zero. (Note: the actual step $\Delta \psi$ used in a scan may be modifled by the varlable RC below)

NP - Number of points on elther side of $(H, K)$ peak. The total number of points $=2 \mathrm{NP}+1$, and is the same for each scan.

If $\mathrm{NP}<1$, the program will 1ssue an error message and return control to the SUPERVISOR.

NB - Number of points (out of total number of folnts $2 \mathrm{NP}+1$ ) on each side of $(\mathrm{H}, \mathrm{K})$ which are used for background purposes to calculate the integrated Intensity of the peak. Total number of background points $=2 \mathrm{NB}$. Note: If the user sets $\mathrm{NB}<1$, then the program automatically sets $\mathrm{NB}=1$. No error message is given. Example with $N P=7, N B=2:$

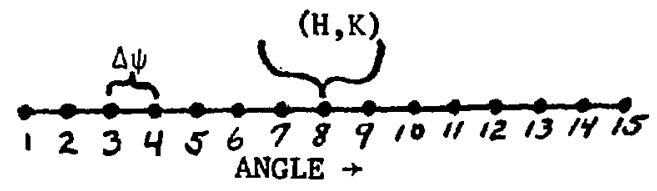

Points $1,2,14$, and 15 determine background. Pt \#8 corresponds to calcliated $(H, K)$ peak position. 
MF - Monitor scale factor. This parameter 1s used only to glve an estimate of the total running time. It should be input in units of kilocounts/ min. e.g., If the monitor counting rate is 200,000 counts/min., then $M F=200$. If $M F=0$ no output is piinted.

RC - Resolution correction. Since the ojserved widths of the peaks vary with scattering angle, RC allows the user to adfust the step size $\Delta \psi$ used in a scan for the resolution of the spectrometer. The algorithm used is

$$
\begin{aligned}
& \Delta \psi=D S
\end{aligned}
$$

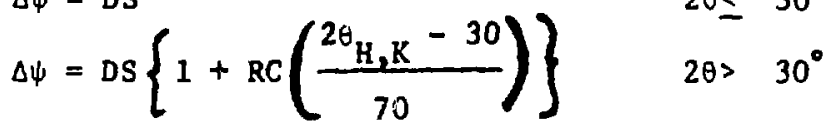

Thus for $R C=1$, the angle step wt1l double from $2 \theta=30$ to $2 \theta=100^{\circ}$. Care is taken to round $\Delta \psi$ so that it is always an integral multiple of $0.01^{\circ}$. For $k_{I} \sim 2.6 \AA^{-1}, R C \sim 1.3$ would be a good cholce; for $k_{1} \sim 4.5$ $4.5 \AA^{-1}, R C \sim 2.5$ (see F1g. 1).

Note: RC has no effect if the scattering arm is frozen.

Preset values for the above vartables are:

$\mathrm{DS}=0.1, \mathrm{NP}=10, \mathrm{ST}=1, \mathrm{NB}=5, \mathrm{MF}=0, \mathrm{RC}=0$.

The procedure for handling the scan varlables in BRP is the same as the triple-axis program. For each scan NS, there are three variables which must be Input:

$$
\begin{aligned}
& H 1-H \\
& K 1-K \\
& M 1-M o n 1 t o r
\end{aligned}
$$

Up to 80 scans can be stored In the computer, and up to 80 scans may be requested at one time for successive execution. 


\section{Execution and Use}

The BRP is entered from the SUPERVISOR by typing LG immediately after the $\$ \$$. The program responds by typing a heading and requests the scan order destred. The scan order may be entered in one of four ways: (1),(1i), and (111) are Identical to the Triple-Axis program. (iv) carrlage return - response is determined by the conditions of previous exit from the BRP. If the previous exit occurred af ter completion of a set of specified scans (normal exit), or if it is the Initlal entrance into the program, an error message is typed and then the program will again request a new scan order. If the previous exit was caused by a CTRL-C character aborting the BRP, then the program w11l continue from its point of interruption. BRP is fully reenterable, and parameters such as background counts, total accumulated counts, etc, will be saved.

Example: Scans 1-10 are to be executed. Sometime during scan \#5, the user returns to the SUPERVISOR by typing CTRL-C. He may then continue in the BRP where he left off by reentering the BRP and hitting when the scan order is requested. The program will finish scan \#5 and then continue on to scans 6-10. During the Interruption, the user may change the scan varlables $\mathrm{H} 1, \mathrm{Kl}$ or $\mathrm{N} 1$ for scans 6-10, zero motors, etc, without affecting scan \#5. However, if the scan vartables for NS - 5 or any of the general read varlables are changed, then this could Invalidate the statistical results that are tabulated at the end of scan 5 (see Output). If the ovprlay has to be loaded again (1.e. kS2), then the BRP is automatically Initialized.

Af ter the scan order is entered, then the only other enquiry is 
HEADING?

At this point the user may insert a heading to be typed at the beginning of each scan. If only a carriage return is entered, then the previous heading wi11 be retajued and used.

Next the program estimates the total running time reeded for the srans requested (unless $M F=0$ ). Then the program types

DRY RUN STARTED

The "dry run" checks the scans that have been requested to see if they are executable. A scan will be skipped (and an error message printed) if

1) $\mathrm{HI}=\mathrm{KI}=0$ or $\mathrm{MI}=0$

2) The scattering triangle will not close for $(B, K)$ (1.e. the center point of the scan).

3) The internal limits of any motor are violated for $, H, K)$. In addition, individual points in a scan will be omftted if the internal limits are violated $\mathrm{fct}$ any motor which is defined. (Note: In the dry run, only the first and last points are checked.)

After the scans have been checked, they will be executed. Changes can be made at any time by hitting a CTRL-C and returning to the SUPERVISOR.

\section{Error Messages}

Upon entering the BRP, a check is made to determine if any of the general variables IM - A3 have nongensical values. If one or more of these varlables have not been properly set, then a self-explanatory error message is typed ar l control is returned to the SUPERVISOR. The BRP checks its own general variables (DS-RC) and types the error message

ILLEGAL DATA

if a problem is found. The following condtitions abort the BRP and return control to the SUPERVISOR: 
1) The sample motor number (C2) is undefined, or the scattering arm motor (A2) is undaftned with ST $\$ 1$, or both. A warning message SAMPLE ANGLE FROZEN and/or SCATTERING ANGLE FROZEN is also typed.

2) ST is not equal to 1,2 , or 3 .

3) NP $<1$

If control is returned to the SUIERVISOR, the user is advised to list the relevant vartables to ascertain the problem.

Other error conditions which cause the ILLEGAL DATA error message to be typed but do not abort the programs are:

1) $H I=K i=0$, or $M I=0$ for some requested scan. Scan will be skipped.

2) The number of scans requested exceeds 80 or is less than 1. A new scan order is requested.

3) An illegal character is inserted in the scan order 1ist. A new scan order is requested.

In addition, if the scattering triangle cannot be satisfied, or if the calculated angles lie uutside the internal motor linits, an error megsage will be typed and the scan or point will be sk1ppeo. 
OUTPUT

At the end of each counting interval the observed angles are typed along with the counts $C_{1}$ and the accumulated sum (SUM). Upon completion of each scan the following information is given:

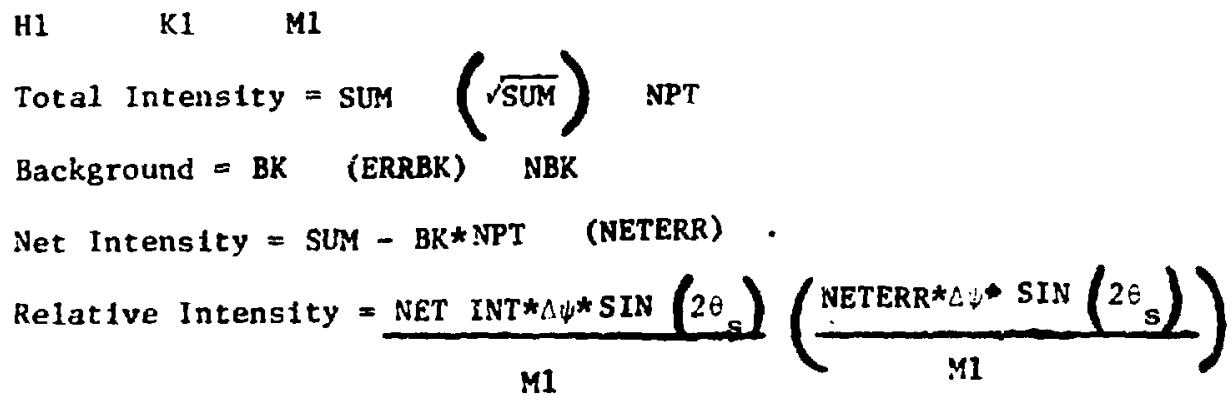

The statistical errors are enclosed in parentineses.

Note: If one or more poincs in a scan are skipped due to a motor limit violation, then the above statistical results are st11l colculated correctly. 
Note: If CTR!-C is hit at the end of a counting interval whlle the ine is being typed, the remainder of the line will not he typed, but $c_{1}$ has already been added to SUM, and if the scan is continued the statistical results wili be correct.

Note: If the scattering arm is frozen, then the Relative lntensity is not corrected for $\sin (20)$ s.

Sample outputs for $S T=1, S T=2$, and $S T=3$ follow. 


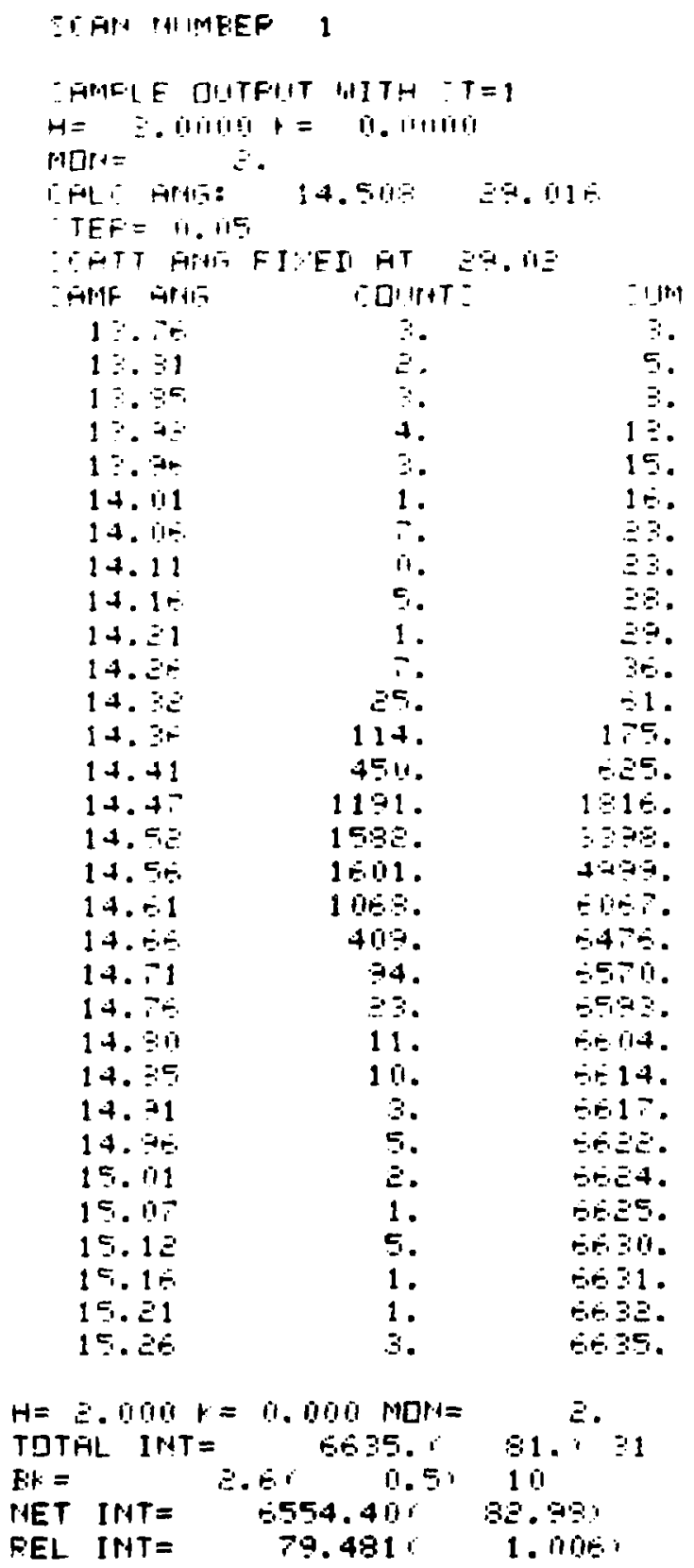




\section{SEFM NIMREF 1}

ZAMPLE OUTFUTT HITH $5 T=\Xi$

$H=E$. Gogin $k=0.0000$

r.TIS =

$$
\text { 三. }
$$

GFLE AHE: 14.5018 39.915

$\because$ TEF $=$ \%. 15

EAMF APH ERATT HNB DQMPTS

$13.55 \quad 27.50$

$15.00 \quad \therefore .50$

13.50 .73

$13.9 \equiv \quad 3.91$

$15.78 \quad 27.95$

14. il $30.0 \mathrm{C}$

14. 17

$14.13 \quad 3.35$

14.1 14.0 .19

$14 . \pm 1 \quad 28.43$

$1+.2=\quad 30.5$

14.

14.510 .75

$14.41 \quad 30.91$

$14.4 \div \quad 23.95$

$14.5 \equiv \quad 5.03$

E. 11

14.5

$1+.51$

14.6 .5

14.71

2.

20.31

29.43

14.78

$1+.80$

50.5

14.85

ㄱ.

14.91

5.7.

14.95

ज曰.

15. 11

59.93

15.

5i. 11

15.11

:1. 11

15.17

$30 . \equiv 1$

15. 51

30.31

30.43

15.8

311.5

E.

$\Xi$.

4.

3.

1.

4.

ii.

1.

1.

+.

5.

$\Xi$.

$1=3$.

515.

$1 \Xi \Xi た 。$

$15=$

125.

$\therefore$.

80.

$P$.

$1 \Xi$.

$E$

$\Xi$

E.

3

1.

1.

4.

$\Xi$

1 .

(i.).

$\therefore$.

4.

$\therefore$

11.

$1 \Xi$

$$
1 \div \text {. }
$$

$1 \therefore$

$1 \%$

$1 \because$.

$\Xi$

三.

5

$1 \div$.

$\therefore 1$.

$1=17$.

ज小.

$4:$ ir.

$5.5+3$

54.

511 .

$50 \%$

5950

5 .

$5=5$.

$5+2$

$c i=i$

$5=34$.

$5+39$.

540.

$5+1$.

E. $\quad 5.243$

$H=E .000 \%=0.000 \mathrm{MDH}=$

TDTFL IPIT= $.44 ., \quad 7 ., \because 1$

$\mathrm{EH}=\quad \Xi .2, \quad 0.5, \quad 10$

NET INT $=5874.80 \mathrm{r} 78.45$

PEL INT = F1.240r i. 351 \% 
ZEAH MIMEEF 1

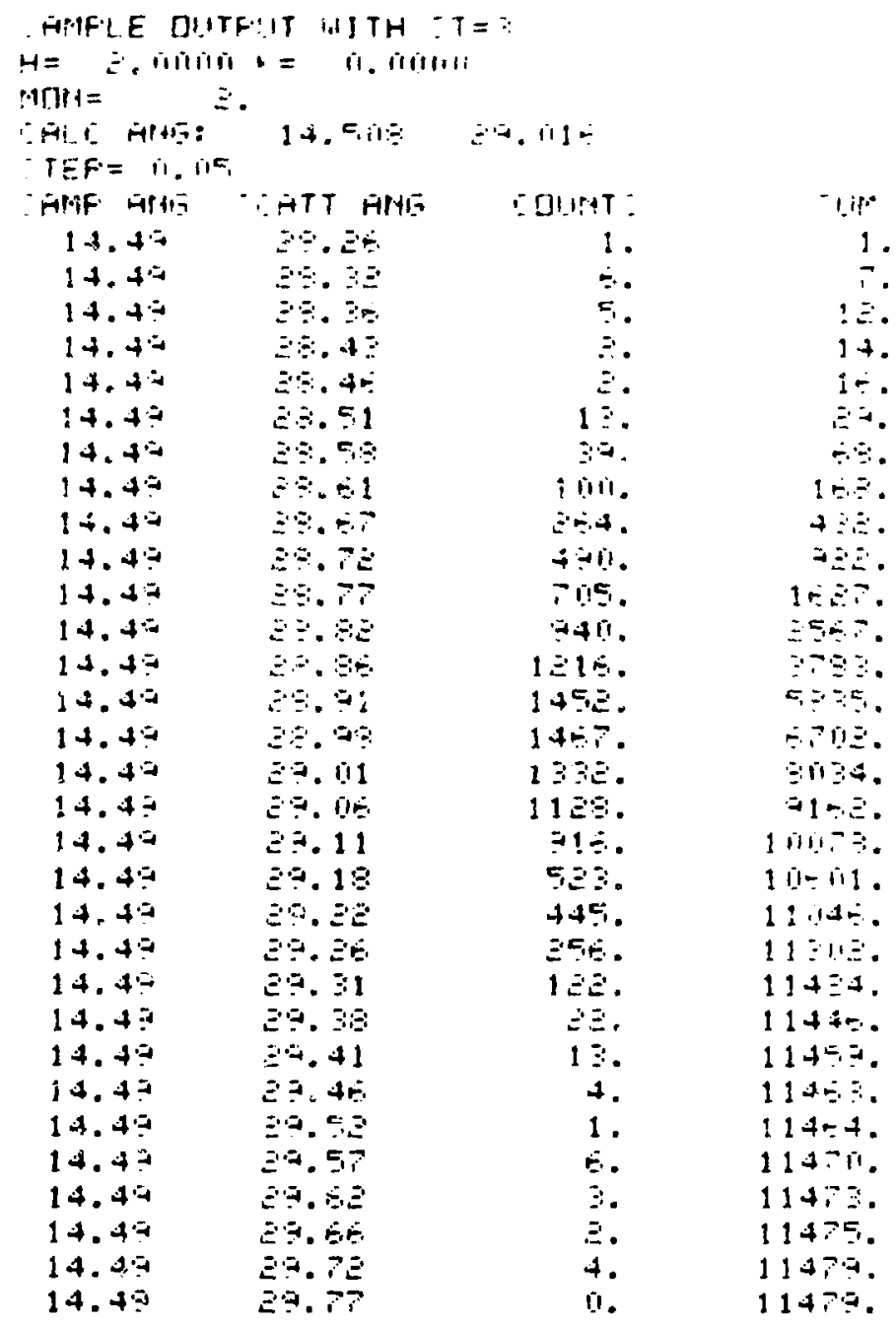

\footnotetext{
$H=E \cdot \operatorname{mog} r=0$. hom $\mathrm{Mar}=$ TOTAL IHT $=11479 . r$ 19\%, $\$ 1$ FE: $=\quad 3.1, \quad$ D. 0.10 MET INT $=11392.70 \% 105.523$ PEL INT $=138.033 \mathrm{r} 1.3151$
} 


\section{READ PACKAGE - Bragg reflection variables}

DS - Step in angle $\Delta \psi$ for sample table when

$S T=1$ or 2 ; step in $2 \theta$ when $S T=3$. (Note: the actual

$\Delta \psi$ used in the scan may be modified by the variable $\mathrm{RC}$ below).

NP - Number of points on either side of $(\mathrm{H}, \mathrm{K})$ peak.

Total number of points $=2 \mathrm{NP}+1$ (same for each scan).

ST - Scan type: 1 for sample rotation scan: 2 for $\theta: 2 \theta$ scan; 3 for

$2 \theta$ scan (with samp angle FROzEN).

NB - Number of points (out of total of 2NP +1 ) on each side of

$(\mathrm{H}, \mathrm{K})$ to be used as background to calculate integrated intensity

of the peak.

MF - Monitor scale factor (in kilocounts/minute). Used only to give an estinate of total running time.

RC - resolution correction. RC allows the user to adjust the step size of each scan to compensate for the change in the resolution of the spectrometer. The step size $\Delta \psi$ is determined by

$$
\Delta \psi=D S \quad 2 \theta \leq 30^{\circ}
$$$$
\Delta \psi=\operatorname{DS}\left\{1+\mathrm{RC}\left(\frac{{ }^{2 \theta} \mathrm{H}_{2} \mathrm{~K}-30}{70}\right)\right\} \quad 2 \theta>0
$$

Scan variables: For each scan NS there are three variables which must be Input: 
HI - H

K1 - K

M1 - Monitor

Up to 80 scans can be stored in the computer, and up to 80 scans

may be requested at one time for successive execution. 


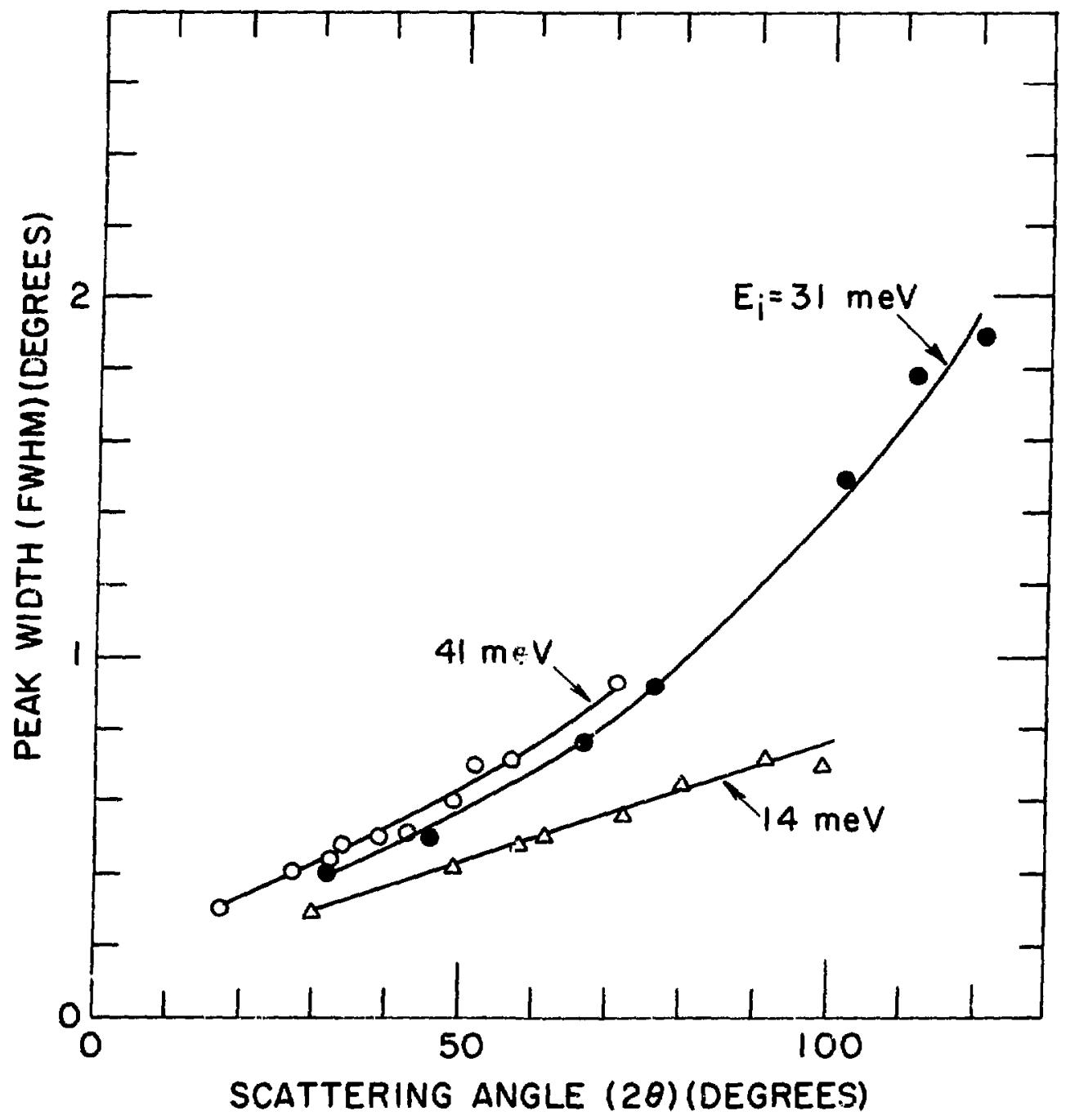

